\title{
Reproductive performance with an automated activity monitoring system versus a synchronized breeding program
}

\author{
R. C. Neves, ${ }^{*}$ K. E. Leslie, ${ }^{\star}$ J. S. Walton, $†$ and S. J. LeBlanc ${ }^{\star 1}$ \\ ${ }^{*}$ Department of Population Medicine, and \\ †Department of Animal and Poultry Science, University of Guelph, Ontario, Canada N1G 2W1
}

\begin{abstract}
The objective was to compare reproductive performance with management programs based on an automated activity monitoring (AAM) system or a synchronized breeding program under field conditions. In total, 1,429 Holstein cows from 3 commercial herds in Ontario, Canada, were enrolled over 1 yr in a randomized controlled trial. At each farm, primiparous and multiparous animals were housed in separate pens. At the pen level, cows were assigned to reproductive management primarily using an AAM system based on monitoring activity levels (Heatime, SCR Engineers Ltd., Netanya, Israel) or a timed artificial insemination (TAI) program. A crossover occurred after 6 mo of the trial to avoid confounding treatment with parity. Insemination based on additional detection of estrus by visual observation was practiced in all pens. At the individual cow level, time to pregnancy throughout the study ( $\mathrm{n}=1,985$ cow- 6 mo periods), time to first service, and time to second service were analyzed with a Cox proportional hazards models accounting for herd, and did not differ overall between the AAM and TAI treatment groups. However, we observed an interaction between herd and breeding program, such that association of AAM- or TAI-based program with these outcomes differed between farms. Time to pregnancy was not different in herd A [median $=151$ and $136 \mathrm{~d}$; hazard ratio $(\mathrm{HR})=0.93]$ and herd $\mathrm{C}($ median $=99$ and $124, \mathrm{HR}=1.24)$, whereas herd $\mathrm{B}$ had a median time to pregnancy of $119 \mathrm{~d}$ and $146 \mathrm{~d}(\mathrm{HR}=1.3)$ in the AAM and TAI groups, respectively. Under conditions in which 19 to $32 \%$ of artificial inseminations in both groups were based on visually detected estrus, herd pregnancy rate and cow-level time to pregnancy did not differ overall between TAI- and AAM-based programs, but the effect of reproductive management approach depended on herd. Considering 924 cow-6 mo periods with artificial inseminations only by the
\end{abstract}

Received December 15, 2011

Accepted June 15, 2012.

${ }^{1}$ Corresponding author: sleblanc@uoguelph.ca assigned program, we also found an interaction between herd and breeding program. Stratified analysis showed no difference in time to pregnancy in herd $\mathrm{A}(\mathrm{HR}=$ $1.3)$, whereas in herds $\mathrm{B}(\mathrm{HR}=1.7)$ and $\mathrm{C}(\mathrm{HR}=2.8)$, cows in the AAM treatment group became pregnant sooner compared with those in the TAI group. Factors that influence the variability in relative performance of these management systems between herds require further investigation. Our study used one commercial activity monitoring system and the results cannot necessarily be generalized to other systems.

Key words: precision technology, estrus detection

\section{INTRODUCTION}

Reproductive performance is very important to dairy herds and is a chronic challenge on most farms. Estrus detection intensity and resulting insemination rate are commonly less than optimal in housed dairy herds, for a variety of reasons (LeBlanc, 2005). This has led to the successful and profitable adoption of systematic timed artificial insemination (TAI) programs in some herds, but many producers still desire to find and breed cows based on estrous behavior. Notwithstanding the efficacy of systematic TAI programs, some producers dislike, or are concerned about the public perception of, the frequent injections required in TAI programs.

Increased activity is recognized as associated with estrus, and automated systems have been developed to detect standing to be mounted or increased activity (Nebel et al., 2000; Firk et al., 2002). Ample evidence exists that activity monitors (pedometers or accelerometers attached to the leg or neck) are able to accurately identify a large proportion of dairy cattle that are in estrus (van Eerdenburg, 2008; Hockey et al., 2010a; Løvendahl and Chagunda, 2010), with a predictable association with the timing of ovulation (Roelofs, 2008; Hockey et al., 2010b). Several studies have described the use of commercially available activity systems and the factors that influence their success (Yániz et al., 2003; López-Gatius et al., 2005), but few studies have measured their performance under commercial conditions or in direct comparison to other tools for manage- 
ment of reproduction. Peralta et al. (2005) used 255 cows in 1 herd over 3 mo in summer to compare estrus detection and conception risks using visual observation, automated detection of being mounted, and activity monitoring. They found no significant differences in probability of estrus detection or pregnancy at AI between visual observation and activity monitoring, and concluded that a combination of at least 2 methods of estrus detection was more effective than any one. Using twice-weekly milk progesterone samples as the gold standard, Holman et al. (2011) used 67 cows to simultaneously compare 2 automated activity monitoring systems, heat-mount detectors, and visual observation. The automated activity monitors had sensitivity of 58 to $63 \%$ for detection of low progesterone periods and 74 to $94 \%$ positive predictive value for signaled heats. The sensitivity of each was improved somewhat when combined with visual observation. A small study comparing a pedometry system and the Ovsynch protocol under Israeli conditions found no differences in reproductive performance (Galon, 2010).

The objective of the present study was to compare herd- and cow-level reproductive performance between reproductive management programs based on an automated activity monitoring system or a TAI program in freestall dairy herds under commercial field conditions. Our hypothesis was that similar overall reproductive performance would be achieved using an activity monitoring system as with a TAI program.

\section{MATERIALS AND METHODS}

A convenience sample of 3 commercial freestall dairy herds located in Ontario, Canada, participated in the study. To detect a difference between 100 and $120 \mathrm{~d}$ in median time to pregnancy with a 180-d follow-up period, 1,398 cows (equally assigned to the 2 reproduction management program treatment groups) were required (Abramson, 2011). Herd inclusion was dependent on the exclusive use of AI and the housing of lactating cows in the breeding period in at least 2 separate pens with similar flooring, size, stabling, and diet. All herds had 4-row barns with concrete floors and attached milking parlors. Herds A, B, and C milked 495, 305, and 260 cows, on average, respectively. Descriptive information on production and management is given in Table 1. All herds had been using a TAI program for the majority of $\mathrm{AI}$ in the lactating cows for several years before this study.

Data were collected from September 2009 until January 2011, during which time each herd was enrolled for 1 yr. All herds housed primiparous cows in a separate pen from multiparous cows. At the pen level, animals were assigned either to an automated activity monitoring (AAM) system (Heatime, SCR Engineers Ltd., Netanya, Israel) or to a TAI program as the primary method of reproductive management for the pen. Study treatments were crossed over after 6 mo of the trial to avoid confounding treatment with parity. The AAM

Table 1. Descriptive information on the study herds in a randomized controlled trial comparing reproductive performance with programs based on automated activity monitoring for estrus detection or a synchronization program for timed AI

\begin{tabular}{|c|c|c|c|}
\hline Descriptor & Herd A & Herd B & Herd C \\
\hline Milking frequency per day & 3 & 2 & 2 \\
\hline Proportion of study animals in lactation $1(\%)$ & 40 & 44 & 40 \\
\hline Study dates & $\begin{array}{l}\text { September } 2009 \text { to } \\
\text { September } 2010\end{array}$ & $\begin{array}{l}\text { October } 2009 \text { to } \\
\text { November } 2010\end{array}$ & $\begin{array}{c}\text { November } 2009 \text { to } \\
\text { December } 2010\end{array}$ \\
\hline $\begin{array}{l}\text { Number of times per day AI was performed based on } \\
\text { AAM }^{1} \text { system alerts }\end{array}$ & Once & Twice & Twice \\
\hline Timed AI program ${ }^{3}$ & $\begin{array}{l}\text { Presynch } 14 / \\
\text { Ovsynch } 56\end{array}$ & Ovsynch 56 & Co-Synch 72 \\
\hline $\begin{array}{l}\text { Mean ( } \pm \text { SEM) DIM at start of the timed AI program } \\
\text { (time of first GnRH) }\end{array}$ & $66 \pm 1$ & $72 \pm 1.5$ & $73 \pm 1$ \\
\hline Stage of pregnancy diagnosis (days after $\mathrm{AI}$ ) & 38 to 45 & 27 to 41 & 28 to 42 \\
\hline Prevalence of lame cows ${ }^{4}(\%)$ & 11 & 25 & $27^{5}$ \\
\hline
\end{tabular}

${ }^{1}$ Automated heat detection based on activity monitoring. Each cow was only inseminated once per signaled estrus.

${ }^{2}$ Voluntary waiting period $=$ minimum days after calving for a cow to be eligible to be bred based on detection of estrus.

${ }^{3}$ Ovsynch $56=$ GnRH-7 d-PGF ${ }_{2 \alpha}-2$ d-GnRH-56 h-TAI; Presynch $14=\mathrm{PGF}_{2 \alpha}-14$ d-PGF ${ }_{2 \alpha}-14$ d-start Ovsynch 56; Co-Synch $72=$ GnRH$7 \mathrm{~d}-\mathrm{PGF}_{2 \alpha}-72 \mathrm{~h}-\mathrm{GnRH}$ and TAI.

${ }^{4}$ Proportion of cows with gait score $\geq 3$ (Sprecher et al., 1997) assessed at the beginning and end of each 6-mo treatment assignment period. ${ }^{5}$ In herd $\mathrm{C}$ only, the proportion of lame cows differed between pens housing primiparous (15\%) and multiparous (39\%) animals, but treatment was not confounded with lameness prevalence. 
system was installed according to the manufacturer's directions, with optical readers for the neck-mounted accelerometer data tags at each entrance to the milking parlor. Therefore, activity data were captured and updated at each milking. Animals in the AAM group were bred based on increased activity. An estrus was signaled when the weighted activity calculated using a proprietary algorithm in the system's software surpassed a set threshold. The exact calculation of the activity score from the commercial software is proprietary and not known to us, but the threshold was essentially 5 standard deviations greater than the average activity of the individual cow in the same period in the past $7 \mathrm{~d}$. The same software and settings were used for all cows in all herds throughout the study.

In addition, all herds continued to breed cows based on visually detected estrus in both treatment groups (i.e., observation of standing to be mounted; secondary signs of estrus may also have been used. Producers did not record details of the signs observed for each AI, but the same personnel, methods, criteria, and intensity of visual observation were used for all cows in all pens within a herd throughout the study.). At the start of a 6-mo AAM treatment period, all nonpregnant animals in the pen received an activity tag collar. To allow for generation of baseline activity, the study period began $7 \mathrm{~d}$ after AAM tags were first applied to a pen. As cows calved and joined the AAM group, they received a collar at calving. The neck collars were kept on for at least $60 \mathrm{~d}$ after confirmation of pregnancy. In herds A and C, multiparous cows were randomly assigned to the AAM system and primiparous animals to TAI-based management for the first half of the study, whereas in herd B the activity system was initially allocated to the firstlactation animals and TAI to the multiparous animals. After $6 \mathrm{mo}$, the treatment assignments were switched between pens (e.g., in herd A, primiparous cows were assigned to the AAM treatment group for the second half of the study). Therefore, each herd was on the study for $12 \mathrm{mo}$, and in all herds, all cows of all parities were included, and treatment was not confounded with parity or with season.

Reproductive practices were discussed at the beginning of the trial with each herd manager to ensure compliance with the protocols during the study period. Descriptive information on reproduction management is given in Table 1. One manager in each of herds B and $\mathrm{C}$ performed the majority of AI, whereas herd A had just over half of the animals in each treatment group bred by a professional AI technician and the remainder by 2 herd managers. All managers had completed formal AI technique training and had been inseminating in their own herds for at least several years. Cows that did not become pregnant in the first $6 \mathrm{mo}$ of the trial were carried over to the second period and therefore, their breeding program was switched. Approximately 31,12 , and 36 different sires were used during the study period in herds $\mathrm{A}, \mathrm{B}$, and $\mathrm{C}$, respectively; bulls were selected by herd owners independent of pen such that sires were used randomly across treatment groups.

All inseminations were individually recorded as being based on the AAM system, the TAI protocol, or visual observation. In all herds, the cows not detected in estrus by the AAM system by 80 DIM or by $45 \mathrm{~d}$ after a previous AI (after diagnosis of nonpregnancy) were allowed to have hormonal treatment to hasten insemination at the discretion of the herd manager and veterinarian. In the majority of cases, the protocol used on those animals was the same TAI synchronization program used for a cow diagnosed open in each herd (i.e., Ovsynch). The AI following hormonal treatment was recorded as a TAI breeding. Lameness prevalence was measured at the pen level using a 5-point scale (Sprecher et al., 1997) at the start of period 1, 6 mo later (cross-over of pen treatment assignments), and at the end of the period 2. Cows were observed while walking back to the stalls from the milking parlor. Animals assigned a gait score of 3 or greater were considered lame. The prevalence of lame animals per pen for each period was average of the proportion lame at the start and end of the study period.

On-farm records, including calving, inseminations, pregnancy, do-not-breed status, and culling dates, were retrieved from DairyComp 305 (Valley Agricultural Software, Tulare, CA) every $60 \mathrm{~d}$. On the same day, a backup of the Heatime software was performed, including inventories of cows and activity tags with confirmation of daily tag data capture. Individual cow data were exported from DairyComp 305 to Microsoft Excel (Microsoft Corp., Richmond, WA). Data editing and statistical analyses were performed with SAS software (version 9.2, SAS Institute Inc., Cary, NC).

\section{Herd-Level Analysis}

Using the command BREDSUM $\backslash \mathrm{E}$ in DairyComp 305 (i.e., pregnancy risk analysis according to 21-d cycle eligibility criteria established by the software), all pregnancies and the total 21-d cow-periods eligible for pregnancies stratified by period of the study and parity (primiparous and multiparous, corresponding to pen housing assignment) were retrieved for each herd. The mean annual 21-d pregnancy risks for the TAI and AAM treatment groups were analyzed, utilizing least squares means controlling for herd as a random effect in a linear mixed model (MIXED procedure). 


\section{Cow-Level Analysis}

Individual cow parity group was categorized into 2 levels (first; second or greater), whereas season of calving and breeding were each categorized into 4 levels (fall: September to November; winter: December to February; spring: March to May; summer: June to August). Because not all animals at the beginning of each study period were in early lactation, and a crossover was performed halfway through the study, time to pregnancy during the study period was the outcome of interest and the unit of analysis was the cow-treatment period. Therefore, cows carrying over from the first to the second period of the trial contributed 2 separate periods. Independence of observations of cows that crossed over periods and treatments was assessed with variance inflation factors (REG procedure in SAS) and was confirmed (variance inflation was 1.2; defined as $<10$ according to Dohoo et al., 2003). Therefore, correlation of cows between study periods was not accounted for further. Because of specific farm factors (e.g., adjustment of group sizes for milking management or to avoid overcrowding), some animals were not assigned to the proper treatment group according to lactation group. Thus, each cow-period was checked for the proper reproductive program, wherein animals assigned to the AAM system but not showing any records of having had an activity tag or an AAM breeding code were assigned to the TAI group. In the same way, cows assigned based on parity to the TAI program but with evidence of having had an activity tag and an AAM breeding code were placed in the AAM group. Herds $\mathrm{A}, \mathrm{B}$, and $\mathrm{C}$ had 92,125 , and 26 cow-treatment periods reassigned according to these criteria, respectively.

Time to Pregnancy. Time to pregnancy during the study period (survival function) was estimated using the Kaplan-Meier method (LIFETEST procedure in SAS). This method was used to compute crude median time to pregnancy and corresponding graphs for AAM and TAI groups. The effect of treatment on time to pregnancy was analyzed using Cox proportional hazards regression (PHREG procedure). In addition to the main effect of treatment, the effect of parity group, season of calving, and period of study (1: first half of the trial; 2: second half of the trial) were offered to the model as covariates. Using backward stepwise selection, all predictors were offered to the model and, at each step, the variable with the largest $P$-value $>0.05$ was deleted. Interactions between treatment and herd, parity, season, and study period were tested. Two approaches were taken to address herd clustering (correla- tion of cows within a herd). Herd was treated as a fixed effect, or as a random effect in which robust standard errors of the main effect estimate were calculated using the robust variance estimator method (COVS option in PHREG) to correct for dependence among the observations within the herds (Allison, 2010). The proportional hazards assumption of the model was assessed using the PH and RESAMPLE options, which uses a Kolmogorov-type supremum test. If a significant $P$-value is obtained, it is indication of poor model fit (Allison, 2010). If the predictor of interest violated the proportional hazards assumption of the model, the product term of the time-dependent covariate (e.g., treatment) and the event time (e.g., days to pregnancy) was forced into the model (Allison, 2010).

Further analysis evaluated time to pregnancy only among the animals that never had an AI based on visual heat detection. Therefore, only cow-periods that had breeding codes based on the assigned reproductive protocol were considered for this analysis. The Cox proportional hazards model building process was the same as above. Study period was the only significant covariate and a significant interaction between herd and treatment was present.

Time to First AI and from First to Second AI. A Cox proportional hazards model similar to that used to assess time to pregnancy was built to evaluate the effect of treatment on time to first service and time from first to second service during the study period. Intervals to AI in days were estimated using the KaplanMeier method (LIFETEST procedure). Analysis of the interval from first to second AI was limited to those within $100 \mathrm{~d}$. The Cox proportional hazards model of time to first service had season of calving and study period as significant covariates so these were retained in the model of time from first to second AI as well. Both models included herd as a fixed effect and a significant interaction between treatment and herd was present.

Conception Risks. A multivariable logistic regression model was built to compare the probability of diagnosed pregnancy for each insemination by breeding code (TAI, AAM, or observed heat), using the GLIMMIX procedure in SAS. An identical approach to the Cox proportional hazards model building process was taken for the determination of the final model. Using pregnancy as the binary outcome of interest, the final model included breeding code as the main effect, accounting for the fixed effects of breeding season, lactation group, and herd. Breeding season and parity group were forced into the model due to a priori concern of their confounding effects. 


\section{RESULTS}

\section{Herd-Level Analysis}

Each treatment group comprised 6 herd-study periods, based on a total of 6,165 (21-d) cow-periods for the overall pregnancy risk analysis across the 3 herds. In crude overall analysis, the mean annual herd $21-\mathrm{d}$ pregnancy risk did not differ $(P=0.25)$ between the TAI-based program (15.9\%) and AAM-based system (14.6\%), but approximately 10 to $>40 \%$ of $\mathrm{AI}$ among treatment groups and farms were not based on the assigned management tool.

\section{Cow-Level Analysis}

In total, 1,429 animals were enrolled, resulting in 1,985 cow- 6 mo periods included in the cow-level analysis. At the start of the study, cows eligible to become pregnant that were past the voluntary waiting period were enrolled in the trial. The mean (SE) DIM was $56.5 \pm 3 \mathrm{~d}$ at the start of the first study period and 56 $\pm 3 \mathrm{~d}$ for the second study period. The proportions of AI within each assigned reproduction management program (AAM or TAI) that were based on that program or visually observed estrus are illustrated in Figure 1. Overall, 19 to $32 \%$ of individual AI events were based on visual observation. Within the AAM treatment group, 11 to $15 \%$ of AI were based on visual observation of estrus, whereas 26 to $44 \%$ of $\mathrm{AI}$ in the TAI-assigned pens were based on observed estrus. Cumulatively, 924 of the 1,985 cow- 6 mo periods did not include any AI based on visual observation.

Time to Pregnancy. In univariable analysis, median time to pregnancy was not different between the AAM $(122 \mathrm{~d})$ and TAI $(137 \mathrm{~d})$ treatment groups $(P=0.19)$. The hazard ratios (HR) for AAM treatment relative to TAI were the same (1.13) for the models treating herd as a fixed effect (95\% CI around the HR: 0.99 to 1.28; $P$ $=0.08)$ or in a cluster-adjusted robust variance method (random herd effect; 95\% CI: 0.94 to $1.37 ; P=0.19$ ) and similarly showed no significant difference between programs. A survival curve of time from enrollment to pregnancy is shown in Figure 2. Lactation group, calving season, and period of study were not significant covariates. The final model with herd as fixed effect demonstrated a significant interaction between herd and treatment, such that the association of reproductive program with time to pregnancy differed between farms. Median times to pregnancy are described in Table 2. The Cox proportional hazard models stratified by herd (Figure 3) revealed no significant difference in time to pregnancy between cows managed in the AAM- based system versus TAI treatment in herd A $(\mathrm{HR}=$ $0.93,95 \%$ CI: 0.75 to $1.15 ; P=0.52)$, a tendency for a for a shorter interval in the AAM group in herd C (HR $=1.24,95 \%$ CI: 0.97 to $1.57 ; P=0.08$ ), and a shorter interval in the AAM group than in the TAI group in herd $\mathrm{B}(\mathrm{HR}=1.3,95 \% \mathrm{CI}: 1.04$ to $1.64 ; P=0.02)$.

The cow-level analysis restricted to the assigned system (AAM or TAI; no visually observed estrus included in the analysis) included 924 cow- 6 mo periods. Overall, univariable median time to pregnancy was 82 d (95\% CI: 75 to 91 d) for AAM and 125 d $(95 \%$ CI: 113 to 175 d) for TAI $(P<0.0001$; Figure 4$)$. Again, we observed a significant interaction between herd and treatment. Median times to pregnancy are described in Table 2. In stratified analysis accounting for study period, we found no difference in time to pregnancy in herd $\mathrm{A}(\mathrm{HR}=1.29,95 \% \mathrm{CI}: 0.91$ to $1.79 ; P=0.12)$, whereas in herds $\mathrm{B}(\mathrm{HR}=1.67,95 \% \mathrm{CI}: 1.2$ to $2.3 ; P$ $=0.002)$ and $\mathrm{C}(\mathrm{HR}=2.78,95 \% \mathrm{CI}: 1.82$ to $4.23 ; P$ $<0.0001)$, cows in the AAM treatment group became pregnant sooner than in the TAI group.

Time to $\boldsymbol{A I}$. Similar to the time to pregnancy model, overall median time to first service $(P=0.3)$

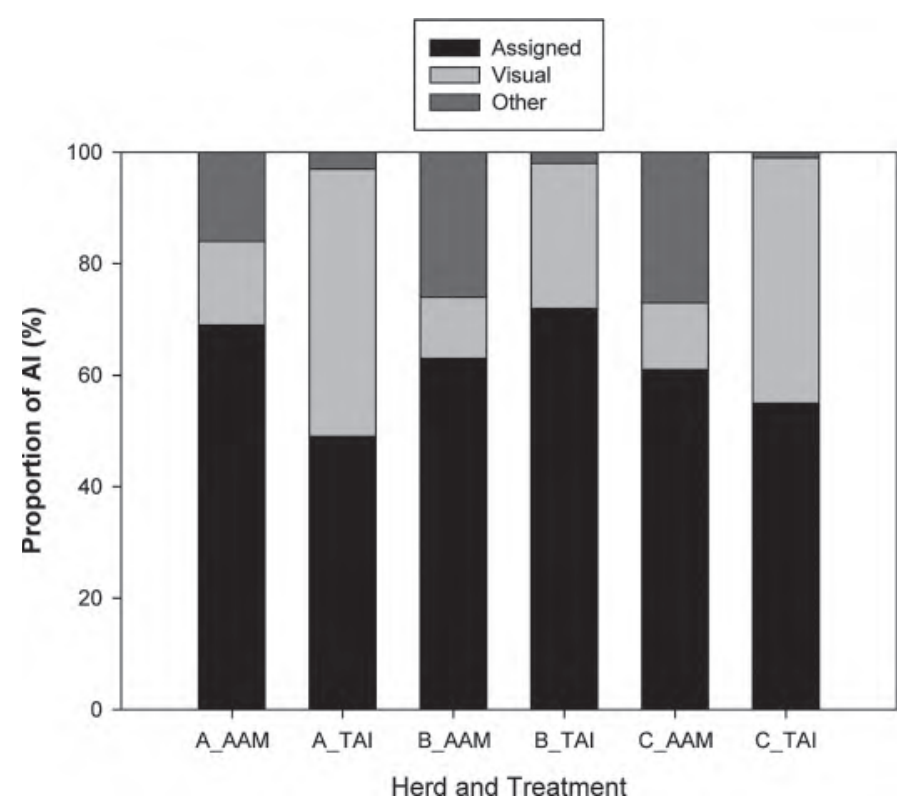

Figure 1. The proportion of inseminations $(\mathrm{n}=3,015)$ that was based on an automated activity monitoring (AAM) system using activity monitoring, a synchronization program for timed AI (TAI), or visual observation of estrus behavior in 3 commercial dairy herds (herds A, B, and C) in each of which 1 pen of cows was assigned to be managed by AAM and 1 pen by TAI. Within the AAM assignment, "Other" includes cows receiving an injection of $\mathrm{PGF}_{2 \alpha}$, an ovulation synchronization treatment, or a progesterone device, and within the TAI assignment, "Other" includes cows that received a progesterone device. 
Table 2. Summary of univariable analyses (95\% CI in parentheses) of times to first insemination and pregnancy in 3 herds in which cows were randomly assigned to reproductive management treatment based on a commercial automated activity monitoring (AAM) system or based on timed AI (TAI) ${ }^{1}$

\begin{tabular}{|c|c|c|c|c|c|}
\hline Herd & Treatment & \multicolumn{2}{|c|}{$\begin{array}{l}\text { All AI, including those based on } \\
\text { visually observed estrus } \\
(\mathrm{n}=1,985 \text { cow- } 6 \text {-mo periods })\end{array}$} & \multicolumn{2}{|c|}{$\begin{array}{l}\text { Only AI based on the assigned } \\
\text { management program } \\
(\mathrm{n}=924 \text { cow- } 6 \text {-mo periods })\end{array}$} \\
\hline \multirow[t]{2}{*}{$\mathrm{A}$} & AAM & $151(124-189)$ & $66(60-70)$ & $78(68-97)$ & $61(52-72)$ \\
\hline & TAI & $136(114-162)$ & $68(59-70)$ & $91(76-136)$ & $71(69-73)$ \\
\hline $\mathrm{C}$ & TAI & $124(108-147)$ & $72(68-75)$ & $120(96-187)$ & $80(77-87)$ \\
\hline
\end{tabular}

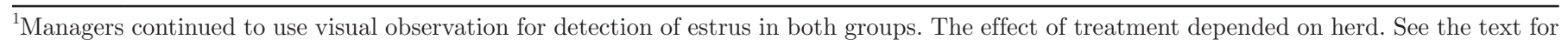
statistical differences between treatments.

${ }^{2}$ Time on the study; not equal to the interval from calving to the outcome.

and time from first to second service $(P=0.2)$ were not different between the AAM and TAI treatment groups. However, an interaction between treatment and herd was significant in both models. Median times to first AI are described in Table 2. The Cox proportional hazards models of time to first AI during the study period stratified by herd revealed no significant difference between cows managed in the AAM-based group and the TAI-based group in herd A (HR $=0.82,95 \% \mathrm{CI}$ : 0.59 to $1.14 ; P=0.24)$ and herd $\mathrm{B}(\mathrm{HR}=0.7,95 \% \mathrm{CI}$ : 0.46 to $1.06 ; P=0.09$ ), whereas in herd $\mathrm{C}$, the median time to first service was shorter in the AAM group than

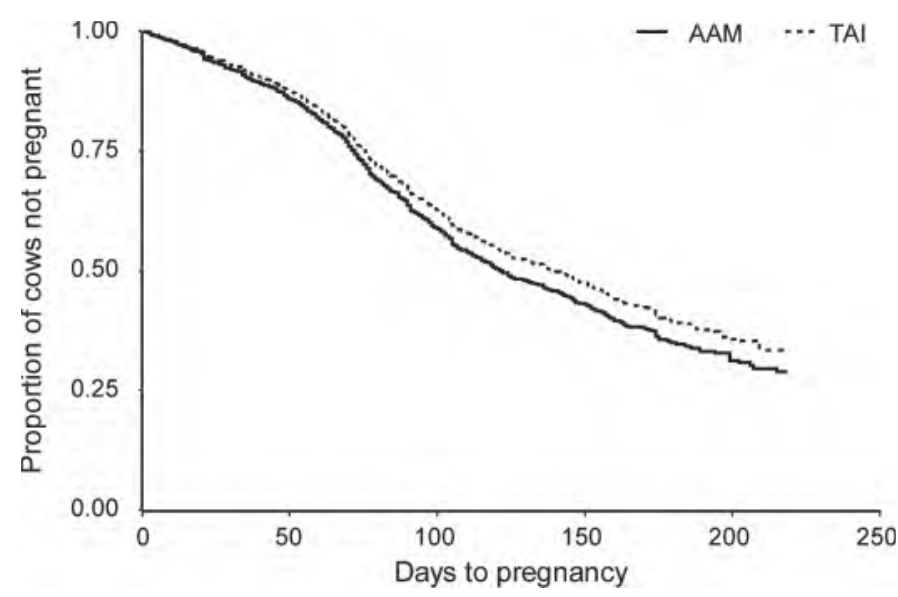

Figure 2. Survival curves for time to pregnancy during the study period ( $\mathrm{n}=1,985$ cow-periods in 3 herds over $1 \mathrm{yr}$ ) for all inseminations. Pens of cows were randomly assigned to reproductive management primary based on automated activity monitoring (AAM; a commercial system using activity monitoring) or based on a synchronization program for fixed-time AI (TAI). Treatment assignments were crossed over after 6 mo to avoid confounding parity with treatment. We observed no overall difference in time to pregnancy but did find a significant interaction of treatment with herd. in the TAI group ( $\mathrm{HR}=2.32,95 \% \mathrm{CI}: 1.53$ to $3.51 ; P$ $<0.0001)$.

The Cox proportional hazards models of time from first AI to second AI stratified by herd indicated a tendency for a shorter interval in herd B for cows managed in the AAM versus TAI system (median $=35$ and $42 \mathrm{~d}$; $\mathrm{HR}=3.30,95 \%$ CI: 0.90 to $12.01 ; P=0.07)$, whereas in herd A (median $=32.5$ and $29 \mathrm{~d} ; \mathrm{HR}=0.53,95 \% \mathrm{CI}$ : 0.30 to $0.93 ; P=0.02$ ) and herd $\mathrm{C}$ (median $=25$ and $35 \mathrm{~d}$; HR $=1.97,95 \%$ CI: 1.45 to $2.67 ; P<0.0001$ ), the intervals between AI were significantly but divergently different. In all analyses, the TAI breeding program was the referent and the hazard ratios correspond to the AAM breeding program.

Conception Risks. The logistic regression model of probability of pregnancy at each insemination included 3,015 AI (1,303, 726, and $986 \mathrm{AI}$ in herds A, B, and $\mathrm{C}$, respectively). We found no difference $(P=0.43)$ in least squares means conception risk (CR) accounting for herd, breeding season, and parity between AI based on AAM (mean, 95\% CI; 31\%, 0.28 to 0.34$)$, TAI (30\%, 0.28 to 0.33$)$, or visual observation of estrus $(33 \%, 0.30$ to 0.36 ). Although $\mathrm{CR}$ varied between herds (herd A $28 \%$, herd B $40 \%$, and herd C 27\%; $P<0.001$ ), the effect of treatment group on $\mathrm{CR}$ did not depend on herd (treatment $\times$ herd interaction, $P=0.66$ ). Overall, we observed a tendency $(P=0.09)$ for differences in CR between herds among AI based on AAM, TAI, and visual observation. When this was explored with models of CR stratified by herd, we found no differences in least squares means CR (accounting for parity and breeding season) for AI based on AAM, TAI, or visual observation in herd A $(27,27$, and $30 \%$, respectively; pairwise $P>0.33)$ or in herd B $(40,47,37 \%$, respectively; pairwise $P>0.15)$, whereas in herd C $(28,32$, and $24 \%$, respectively; overall $P=0.08$ ), only a differ- 

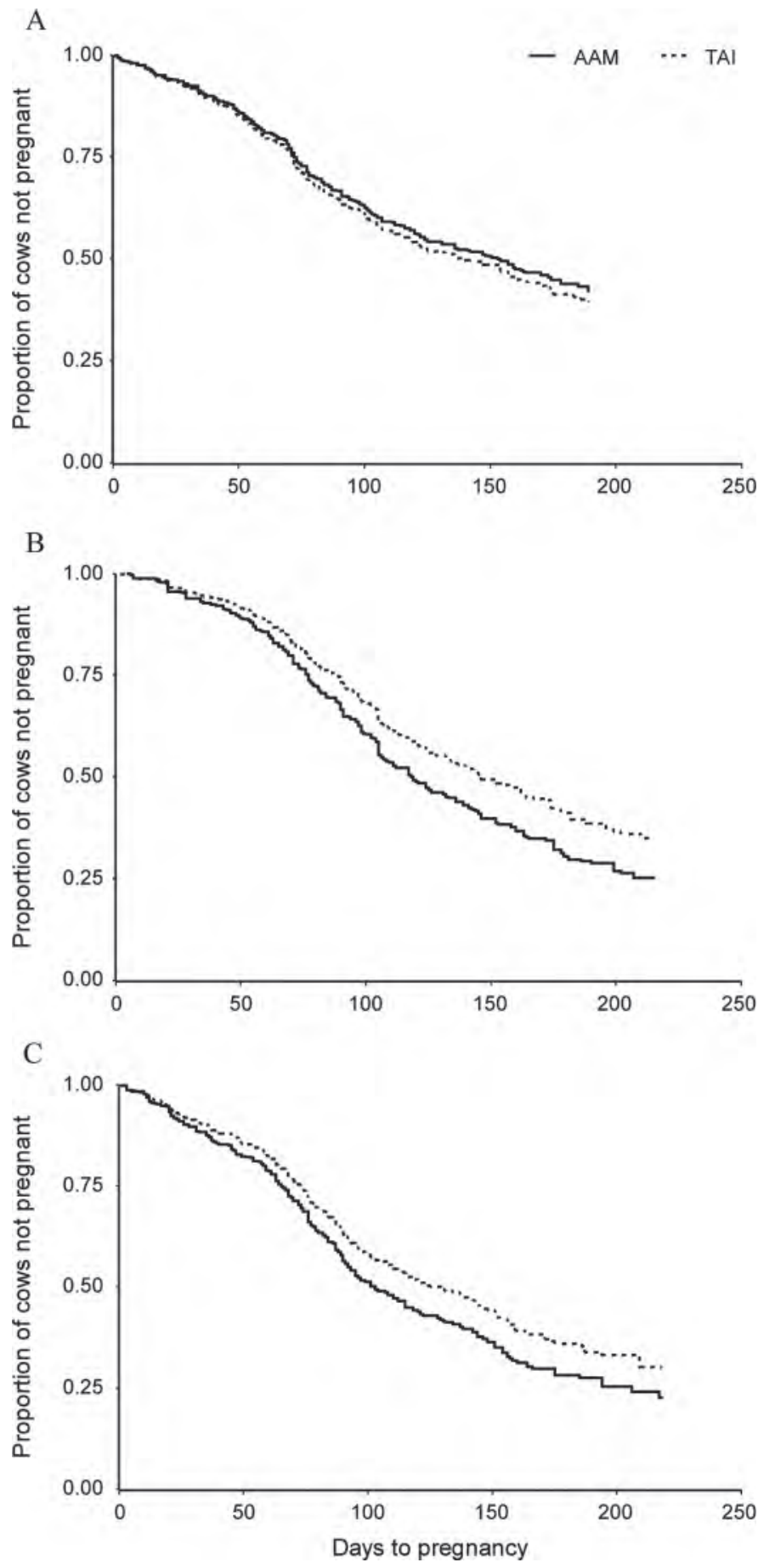

Figure 3. Survival curves for time to pregnancy stratified by herd for herd A ( $\mathrm{n}=808$ cow-periods; panel A), herd B ( $\mathrm{n}=629$ cowperiods, panel B), and herd $\mathrm{C}(\mathrm{n}=548$ cow-periods; panel $\mathrm{C})$. Pens of cows were randomly assigned to reproductive management primary based on automated heat detection (AAM; a commercial system using activity monitoring) or based on a synchronization program for fixedtime AI (TAI). Treatment assignments were crossed over after 6 mo to avoid confounding parity with treatment.

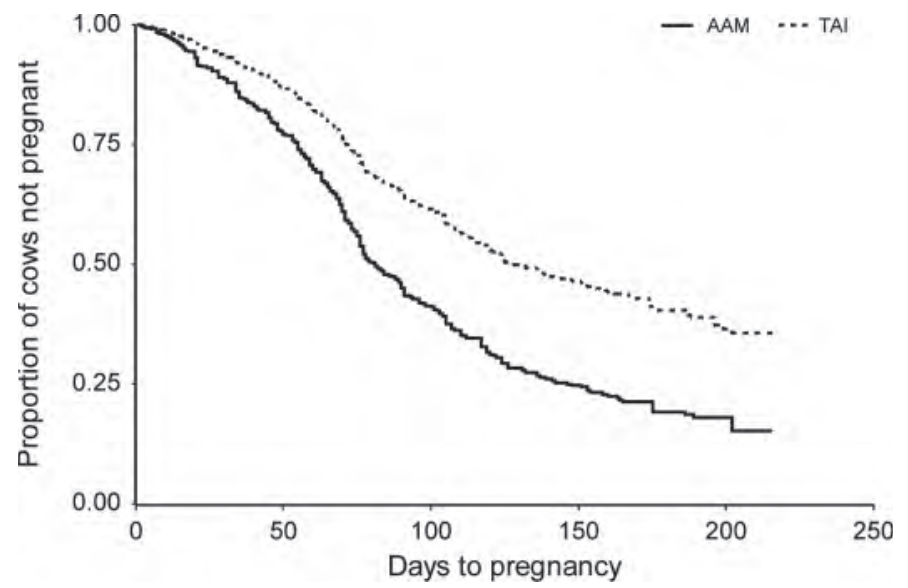

Figure 4. Survival curves for time to pregnancy during the study period including only inseminations based on the assignment treatment program $(\mathrm{n}=924$ cow-periods in 3 herds over $1 \mathrm{yr})$. Pens of cows were randomly assigned to reproductive management primary based on automated activity monitoring (AAM; a commercial system using activity monitoring) or based on a synchronization program for fixedtime AI (TAI). Treatment assignments were crossed over after 6 mo to avoid confounding parity with treatment.

ence between AI based on TAI and visual observation $(P=0.02)$ was found.

\section{DISCUSSION}

The present study is novel in comparing the longterm reproductive performance of commercial dairy herds managed with an AAM system relative to a TAI-based program under North American housing and management conditions. The main finding was that overall reproductive performance was similar between management based on the AAM system and a synchronization program for TAI, but differences did exist between herds in relative performance with these 2 management tools, which varied from no significant difference to substantially shorter times to pregnancy with the AAM system. This was especially apparent in the analysis restricted to AI based only on the AAM system or on TAI. Some strengths of this study are that it assessed an economically important outcome with randomized assignment to treatment in multiple commercial herds over a full year. Conversely, use of large numbers of cows in commercial herds required acceptance of some differences between herds in the specifics of the TAI programs and inevitably in other aspects of management that could confound the effects of the reproductive program treatments. Additionally, this was designed as a cow-level study but the results suggest that herd-level variables may influence the results. The interactions of treatment with herd highlight the need for additional studies to probe the factors that 
influence relative results obtained with an AAM or TAI program. However, it would be a substantial challenge to conduct a randomized controlled study with a sufficient number of herds to properly investigate herd-level variables that affect the performance of AAM as management tool or to compare the performance of AAM to TAI or other management options, using pregnancy outcomes over a full year.

The primary objective was to measure and compare AAM and TAI as management tools as they are implemented and used under field conditions, not necessarily to the exclusion of all other tools (i.e., estrus detection through visual observation). We included the herd annual 21-d pregnancy risks for each group as this is a widely used, overall measurement of herd reproductive performance. However, we caution that based on the design of this study and the interactions of herd with treatment for the time to pregnancy outcomes, the cow-level analyses more fully describe the results. Differences in reproductive performance between AAM and TAI might have been blurred by the use of visual detection of estrus. To address the question of relative performance of these tools if they were used exclusively, we analyzed time to pregnancy considering only cows that had been bred exclusively by their assigned method. In this analysis, time to pregnancy was shorter overall with AAM, with a similar interaction of treatment with herd and similar directions of results (no significant difference in one herd; shorter time to pregnancy in the others), albeit with greater differences between treatments in time to pregnancy when AI based on visual observation were not considered. Insemination of cows in either treatment group that were observed in estrus could create a bias in the remaining population, such that those in obvious estrus might be the most fertile, leaving a group that included anovular, lame, or otherwise less-fertile cows to be detected by the AAM system or inseminated with TAI. However, the present results do not indicate this selection bias because we found no differences in CR between assigned treatment groups or among AI based on AAM, TAI, or visual observation (with the exception of a difference in CR favoring TAI over visual in one herd). Before the start of the study, we speculated that, relative to TAI or visual observation with typically low estrus detection rates, the AAM system might be associated with increased insemination rate (due to the possibility of estrus detection throughout every day) but possibly reduced CR (if, based on activity monitoring, the timings of AI relative to ovulation were less than optimally precise). We found no overall differences between the AAM and TAI assigned groups in times to AI (although variation in effects did exist between herds) and no differences in CR between AI based on AAM or other means.

The study herds did not use the same synchronization programs for TAI, nor did any of them use their TAI program in a way that might be expected to achieve the shortest time to pregnancy, assuming predominant or exclusive reliance on the TAI program. Specifically, systematic use of a synchronization program with cohorts of cows enrolled weekly, use of a program that appears to increase $\mathrm{CR}$ to the maximum currently attainable in an intensive, confined production system (e.g., "Double Ovsynch"; Souza et al., 2008), and systematic resynchronization of nonpregnant cows approximately 28 to $32 \mathrm{~d}$ after the previous TAI may be expected to optimize pregnancy rate with this management system. However, in the first instance, TAI programs increase insemination rate by establishing upper limits on times to AI, and they may optimize follicular status to increase CR. Their purpose is not necessarily to eliminate estrus detection, and whether estrus detection is complementary or detrimental to a TAI program depends on estrus detection accuracy and CR (Bilby and Chebel, 2011). The herds in this study used a combination of reproduction management tools, which appears to be representative of many herds in North America (Caraviello et al., 2006). However, differences in TAI programs among herds in the present study may have contributed to the interaction of treatment (AAM or TAI-based management) with herd. Herd A used a Presynch 14/Ovsynch 56 protocol. Presynchronization with $\mathrm{PGF}_{2 \alpha}$ before the start of the TAI program may or may not lead to higher CR compared with cows starting a TAI program at random stages of the estrus cycle (Moreira et al., 2001; El-Zarkouny et al., 2004; Stevenson, 2011). Herd B was on Ovsynch, whereas herd C used a Co-Synch program. Brusveen et al. (2008) found higher CR when lactating dairy cows were synchronized with Ovsynch 56 compared with Co-Synch 72 or Co-Synch 48. In any case, treatment in this trial was randomized within herd, which would minimize bias of the estimate of the overall effect of treatment.

In the present study, 26 to $44 \%$ of AI in the groups assigned to management with TAI were based on visual observation. During a single Ovsynch treatment, up to $15 \%$ of cows will not have a successfully synchronized ovulation (Bilby and Chebel, 2011) and so may come into estrus during the program. If CR were $40 \%$ following TAI, many of the nonpregnant cows would be expected to express estrus approximately 18 to $24 \mathrm{~d}$ later. Therefore, if estrus detection efficiency were $35 \%$, over approximately $5 \mathrm{wk}$ (from the start of a 10-d TAI protocol to $24 \mathrm{~d}$ after AI), approximately $25 \%$ of cows enrolled may be detected in estrus. Although all of the 
study herds had been using a TAI program as their primary reproduction management tool for several years before the start of the present trial, in all herds cows were observed for detection of estrus several times daily before and throughout the study. Among the groups assigned to management with the AAM system, 11 to $15 \%$ of AI were based on visual observation. These likely represent a combination of cows observed to be standing to be mounted or displaying secondary signs of estrus either in the absence of increased activity or before an increase was signaled by the system or read by the manager. A proportion of these AI recorded as being based on visual detection in the AAM treatment groups (i.e., apparently missed by the AAM system) might have preceded a signal from the AAM system by $<12 \mathrm{~h}$; therefore, being a function of time lag to the next milking to capture or looking at the data from the AAM system, rather than failure of the system to detect the estrus event. We were not able to capture and analyze detailed data from the proprietary AAM software system to quantify the occurrence of this scenario. On the other hand, it is likely that some cows that were in estrus were not detected by the AAM system. We did not collect data to determine the accuracy of estrus detection (e.g., measurement of progesterone) by the AAM system or by visual observation relative to behavioral expression or ovulation but the objective was to compare pregnancy rates between AAM and TAI.

Although herds A and C had poor overall CR (28\%) and herd C reasonably good overall CR (40\%), CR did not differ between AI based on AAM and that based on either TAI or visual observation in any herd. Despite their similarly low $\mathrm{CR}$, herd A had no difference in reproductive performance between AAM- and TAIbased programs, whereas herd $\mathrm{C}$ showed the greatest difference in pregnancy rate (hazard ratio) between the treatments. Again, the reasons for these apparent differences between herds are unclear, likely multifactorial, and require further investigation. The fact that herd A conducted pregnancy diagnosis weekly and herds B and $\mathrm{C}$ every two weeks may have favored the apparent performance of the AAM system in herds B and C by allowing more time over which AAM might detect cows in estrus. On the other hand, pregnancy diagnosis was up to $10 \mathrm{~d}$ earlier in herds $\mathrm{B}$ and $\mathrm{C}$ than in herd $\mathrm{A}$, which would allow earlier re-insemination in the TAI group. We did not collect data on the fertility index (i.e., daughter pregnancy rate) of the bulls used for AI during this study, but bulls were selected for each AI within each herd without regard to treatment group, so possible differences in fertility among the many bulls used were not confounded with treatment. The space allowance per cow (degree of stocking or crowding), characteristics of the floor surface in cow traffic areas, degree of competition for access to feed and stalls, and use of heat abatement measures are likely fruitful herdlevel variables for study.

In the present study, herd $\mathrm{C}$ had a notably higher prevalence of lameness among multiparous animals, but this would not have confounded the effect of treatment because treatments were crossed over at the pen level to balance parity distribution within each treatment. Study period was almost completely correlated with parity but neither variable was associated with time to pregnancy. When the analysis was stratified by period of the study (i.e., by parity group), overall or within herd $\mathrm{C}$, the difference in time to pregnancy between the TAI and AAM programs was similar for periods 1 and 2.

Lameness is clearly undesirable for all cows but might be expected to differentially impair reproduction if insemination depends on expression of estrus through increased walking activity. An experimental study reported significantly reduced standing to be mounted activity in lame cows compared with normal animals when in estrus (Sood and Nanda, 2006). The same study reported a considerable increase in estrus behavior score when more than one animal was in estrus in the nonlame cows but the same did not occur with the lame animals. Roelofs et al. (2005) described a greater chance of estrus detection by a pedometer when behavioral estrus was more intense. Garbarino et al. (2004) reported that cows classified as lame were at 3.5 times greater risk of delayed cyclicity during the first $60 \mathrm{~d}$ postpartum compared with nonlame animals. Unintuitively, the herds (B and C) in which the AAM system tended to be or was associated with shorter time to pregnancy had higher prevalence of lameness than in herd A. This suggests that lameness prevalence alone may not determine the performance of an AAM system, but supports the need for further research into the factors, including lameness, that influence the relative success of reproductive management tools and systems among cows and herds.

Across a variety of housing and management systems (e.g., pasture or slatted floors, robotic milking) the interval from onset of estrus based on activity (i.e., time that activity surpassed the system threshold) to ovulation was 29 to $33 \mathrm{~h}$ (Roelofs et al., 2005; Hockey et al., 2010a,b; Yoshioka et al., 2010). Several of these studies used a low activity alert threshold. Hockey et al. (2010a,b) defined an estrus as being an increase of $2 \mathrm{SD}$ of the activity recorded in 2-h intervals for the corresponding periods at the same times of the day in the previous $10 \mathrm{~d}$. The definition of estrus onset with the system used in the present study was an increase of $5 \mathrm{SD}$ of the mean activity for the 7 preceding days. Therefore, it is understandable that when a higher ac- 
tivity threshold is used, the expected time from onset of estrus (according to the activity monitor) to ovulation may be reduced. The highest percentage of good quality embryos was obtained when AI occurred 12 to $16 \mathrm{~h}$ before ovulation (Roelofs et al., 2006). Consistent with that, the probability of pregnancy was calculated to be optimal at approximately $12 \mathrm{~h}$ after the onset of estrus as signaled by a pedometer (Maatje et al., 1997). In contrast, Hockey et al. (2010b) found that the probability of pregnancy was greatest at 24 to $40 \mathrm{~h}$ after onset of increased pedometer activity. The system used in the present study has shown optimum probability of pregnancy when cows were inseminated 0 to $16 \mathrm{~h}$ after the peak of the estrus activity (Bar, 2010). Therefore, a greater proportion of AI may fall within the optimal interval if AI happens twice a day rather than once a day, even though an estrus alert is signaled based on the onset of increased activity and not the peak. It is worth noting that although activity or pedometry systems typically collect and present data in 1- or 2-h increments, depending on how often these data are collected or transmitted (e.g., continuously or at milking times), and how often the data for cows that surpass the threshold for estrus are looked at and acted upon by herd managers (typically once or twice a day), the interval from onset of increased activity to AI or from peak activity to AI may be confounded by management practices. Unfortunately, in the present study, detailed data were not collected on the timing of insemination relative to the onset or peak of increased activity. Herds $\mathrm{B}$ and $\mathrm{C}$ inseminated based on the AAM system twice a day, which may have improved the timing of AI relative to the onset of increased activity. Conversely, once-aday AI might have contributed to the lack of difference in time to pregnancy between the 2 breeding programs in herd $\mathrm{A}$.

If similar or better reproductive performance can be achieved using an AAM system than with a TAI-based program, that could facilitate genetic selection for expression of estrus as well as for the achievement and timing of pregnancy.

\section{CONCLUSIONS}

When used in combination with visual observation for detection of estrus, overall reproductive performance was not different between the TAI and AAMbased reproduction management systems, but we did observe differences in relative performance between herds. Time to pregnancy was not different or was shorter with an automated activity system relative to a TAI-based program when 19 to $32 \%$ of total AI were based on observed estrus. As in the overall analysis, the effect of management program depended on herd, with time to pregnancy being shorter in 2 of 3 herds, but when considering only AI based on AAM or TAI (excluding cows with visually observed estrus), the differences between treatments were greater. Further research is needed to better understand the herd- or cow-level variables that influence herd reproductive performance with automated estrus detection systems, and their expected performance relative to other tools for herd reproduction management such as systematic TAI programs. Our study used one commercial activity monitoring system and the results cannot necessarily be generalized to other systems. Our results indicate that AAM systems may yield reproductive performance at least comparable to that of a TAI-based program under field conditions but that relative performance may vary moderately between herds.

\section{ACKNOWLEDGMENTS}

This project was funded by the Ontario Ministry of Agriculture and Rural Affairs (Guelph, ON, Canada), Semex (Madison, WI), and SCR Engineers Ltd. (Netanya, Israel). We are grateful to the participating herd owners for their time and interest.

\section{REFERENCES}

Abramson, J. H. 2011. WINPEPI updated: Computer programs for epidemiologists, and their teaching potential. Epidemiol. Perspect. Innov. 8:1.

Allison, P. D. 2010. Survival Analysis Using SAS: A Practical Guide. 2nd ed. SAS Institute Inc., Cary, NC.

Bar, D. 2010. Optimal timing of insemination using activity collars. Page 100 in Proc. First North Am. Conf. Precision Dairy Management, Toronto, Canada. Progressive Dairy Operators, Elora, ON, Canada.

Bilby, T. R., and R. C. Chebel. 2011. Integration of synchronization programs and estrous detection. Pages $32-40$ in Proc. Dairy Cattle Reprod. Conf., Kansas City, MO. Dairy Cattle Reproduction Council, Hartland, WI.

Brusveen, D. J., A. P. Cunha, C. D. Silva, P. M. Cunha, R. A. Sterry, E. P. B. Silva, J. N. Guenther, and M. C. Wiltbank. 2008. Altering the time of the second gonadotropin-releasing hormone injection and artificial insemination (AI) during Ovsynch affects pregnancies per AI in lactating dairy cows. J. Dairy Sci. 91:1044-1052.

Caraviello, D. Z., K. A. Weigel, P. M. Fricke, M. C. Wiltbank, M. J. Florent, N. B. Cook, K. V. Nordlund, N. R. Zwald, and C. L. Rawson. 2006. Survey of management practices on reproductive performance of dairy cattle on large US commercial farms. J. Dairy Sci. 89:4723-4735.

Dohoo, I., W. Martin, and H. Stryhn. 2003. Veterinary Epidemiologic Research. AVC Inc., Charlottetown, PEI, Canada.

El-Zarkouny, S. Z., J. A. Cartmill, B. A. Hensley, and J. S. Stevenson. 2004. Pregnancy in dairy cows after synchronized ovulation regimens with or without presynchronization and progesterone. J. Dairy Sci. 87:1024-1037.

Firk, R., E. Stamer, W. Junge, and J. Krieter. 2002. Automation of oestrus detection in dairy cows: A review. Livest. Prod. Sci. $75: 219-232$.

Galon, N. 2010. The use of pedometry for estrus detection in dairy cows in Israel. J. Reprod. Dev. 56(Suppl.):S48-S52. 
Garbarino, E. J., J. A. Hernandez, J. K. Shearer, C. A. Risco, and W. W. Thatcher. 2004. Effect of lameness on ovarian activity in postpartum Holstein cows. J. Dairy Sci. 87:4123-4131.

Hockey, C., J. Morton, S. Norman, and M. McGowan. 2010a. Evaluation of a neck mounted 2-hourly activity meter system for detecting cows about to ovulate in two paddock-based Australian dairy herds. Reprod. Domest. Anim. 45:e107-e117.

Hockey, C. D., J. M. Morton, S. T. Norman, and M. R. McGowan. 2010b. Improved prediction of ovulation time may increase pregnancy rates to artificial insemination in lactating dairy cattle. Reprod. Domest. Anim. 45:e239-e248.

Holman, A., J. Thompson, J. E. Routly, J. Cameron, D. N. Jones, D. Grove-White, R. F. Smith, and H. Dobson. 2011. Comparison of oestrus detection methods in dairy cattle. Vet. Rec. 169:47-52.

LeBlanc, S. 2005. Overall reproductive performance of Canadian dairy cows: Challenges we are facing. Adv. Dairy Technol. 17:137-157.

López-Gatius, F. P., P. Santolaria, I. Mundet, and J. L. Yaniz. 2005 Walking activity at estrus and subsequent fertility in dairy cows. Theriogenology 63:1419-1429.

Løvendahl, P., and M. G. G. Chagunda. 2010. On the use of physical activity monitoring for estrus detection in dairy cows. J. Dairy Sci. 93:249-259.

Maatje, K., S. H. Loeffler, and B. Engel. 1997. Predicting optimal time of insemination in cows that show visual signs of estrus by estimating onset of estrus with pedometers. J. Dairy Sci. 80:1098-1105.

Moreira, F., C. Orlandi, C. A. Risco, R. Mattos, F. Lopes, and W. W. Thatcher. 2001. Effects of presynchronization and bovine somatotropin on pregnancy rates to a timed artificial insemination protocol in lactating dairy cows. J. Dairy Sci. 84:1646-1659.

Nebel, R. L., M. G. Dransfield, S. M. Jobst, and J. H. Bame. 2000. Automated electronic systems for the detection of oestrus and timing of AI in cattle. Anim. Reprod. Sci. 60-61:713-723.
Peralta, O. A., R. E. Pearson, and R. L. Nebel. 2005. Comparison of three estrus detection systems during summer in a large commercial dairy herd. Anim. Reprod. Sci. 87:59-72.

Roelofs, J. B. 2008. Prediction of ovulation and optimal insemination interval. Vet. Q. 30(Suppl.1):58-78.

Roelofs, J. B., E. A. M. Graat, E. Mullaart, N. M. Soede, W. Voskamp-Harkema, and B. Kemp. 2006. Effects of insemination-ovulation interval on fertilization rates and embryo characteristics in dairy cattle. Theriogenology 66:2173-2181.

Roelofs, J. B., F. J. C. M. van Eerdenburg, N. M. Soede, and B. Kemp. 2005. Pedometer readings for estrous detection and as predictor for time of ovulation in dairy cattle. Theriogenology 64:1690-1703.

Sood, P., and A. S. Nanda. 2006. Effect of lameness on estrous behavior in crossbred cows. Theriogenology 66:1375-1380.

Souza, A. H., H. Ayres, R. M. Ferreira, and M. C. Wiltbank. 2008. A new presynchronization system (Double-Ovsynch) increases fertility at first postpartum timed AI in lactating dairy cows. Theriogenology 70:208-215.

Sprecher, D. J., D. E. Hostetler, and J. B. Kaneene. 1997. A lameness scoring system that uses posture and gait to predict dairy cattle reproductive performance. Theriogenology 47:1179-1187.

Stevenson, J. S. 2011. Alternative programs to presynchronize estrous cycles in dairy cattle before a timed artificial insemination program. J. Dairy Sci. 94:205-217.

van Eerdenburg, F. J. C. M. 2008. The pedometer, an automated aid in the detection of estrus. Vet. Q. 30(Suppl. 1):49-57.

Yániz, J., P. Santolaria, and F. Lopez-Gatius. 2003. Relationship between fertility and the walking activity of cows at oestrus. Vet. Rec. 152:239-240.

Yoshioka, H., M. Ito, and Y. Tanimoto. 2010. Effectiveness of a realtime radiotelemetric pedometer for estrus detection and insemination in Japanese black cows. J. Reprod. Dev. 56:351-355. 